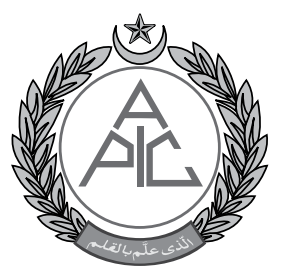

\title{
Continuous renal replacement therapy: understanding the foundations applied to pediatric patients
}

\author{
Evelyn Obando ${ }^{1}$, Eliana López ${ }^{2}$, David Montoya ${ }^{1}$, \\ Jaime Fernández-Sarmiento ${ }^{3}$
}

\begin{abstract}
Continuous renal replacement therapy (CRRT) is a well-established supportive treatment for acute kidney injury in pediatric intensive care units. Knowing its basic aspects allows a rational approach to therapy, making this therapeutic option a more adaptable treatment for individual patient. Different strategies may be used in the same child, depending on the clinical situation and the changes that may present throughout the clinical course. This article explains the physical principles, modalities of continuous renal replacement therapies, and membrane and filter characteristics in order to better understand the transmembrane transport of fluids and solutes in continuous renal replacement therapy.
\end{abstract}

${ }^{1}$ Fellow, Pediatric Intensive Care. Universidad de la Sabana ${ }^{2}$ Fellow, Pediatric Intensive Care. Universidad Nacional ${ }^{3}$ Department of Critical Care Medicine and Pediatrics, Fundación CardioinfantilInstituto de Cardiología. Professor at Universidad de la Sabana. Bogotá, Colombia.

Correspondence:

Jaime Fernández-Sarmiento, MD. Universidad de La Sabana, Campus Universitario del Puente del Común, Km 7 Autopista Norte de Bogotá, Chía - Cundinamarca - ColombiaSuramérica;

Phone: +057-16672727 ext 22; E-mail: JaimeFe@unisabana. edu.co

Received: 6 Jan, 23 Jan 2018

Reviewed: 24 Jan, 17 Feb 2018

Key words: Acute kidney injury; Renal replacement therapy; Continuous renal replacement therapy; CRRT; Hemofiltration; Dialysis; Children; pediatrics

Abbreviations: CRRT = Continuous renal replacement therapy; SCUF = Slow continuous ultrafiltration therapies; FF = Filtration fraction; $\mathrm{CVVH}=$ Continuous venovenous hemofiltration; $\mathrm{AKI}=$ Acute kidney injury; CVVHD = Continuous venovenous hemodialysis; $C V V H D F=$ Continuous venovenous hemodiafiltration; SLEDD = Sustained low-efficiency daily dialysis, EDDf = Extended daily dialysis with filtration, PDIRRT = Prolonged daily intermittent renal replacement therapy

Citation: Obando E, López E, Montoya D, Fernández-Sarmiento J. Continuous renal replacement therapy: understanding the foundations applied to pediatric patients. Anaesth Pain \& Intensive Care 2018;22 Suppl 1:S39-S45

\section{INTRODUCTION}

Acute kidney injury is a frequent comorbidity in patients admitted to the intensive care units. Thirty to $40 \%$ children may be affected and it has an associated mortality of up to $50 \%$.It is considered to be an independent risk factor for mortality in critical care. Approximately $5-10 \%$ of patients with acute kidney injury need renal replacement therapy (CRRT). ${ }^{1}$ In contrast to adult population, children may need CRRT in conditions other than renal failure, such as after cardiac surgery or to remove toxins associated with inborn errors of metabolism. ${ }^{2}$ It is estimated that over $90 \%$ of the pediatric population received CRRT to treat metabolic or fluid abnormalities directly related to acute kidney injury (AKI) such as fluid overload, hyperkalemia, symptomatic uremia and profound metabolic acidosis. Of the remaining patients, $4 \%$ received CRRT to treat hyper ammonemia and $2 \%$ received CRRT to treat an intoxication or medication overdose.

Understanding the basic aspects of CRRTs allows into be used appropriately, at the proper time, and with the lowest possible rate of complications. The objective of this article is to review the important aspects of these types of interventions, beginning with basic concepts and reviewing some variables 
which are important in CRRT.

\section{CONTINUOUS RENAL REPLACEMENT THERAPY (CRRT)}

CRRT is an extracorporeal therapy to filter substances using a semipermeable membrane, which substitutes kidney function, and is applied for 24 hours. It provides slow removal of solutes and solvent which allows more controlled management of the unstable or potentially unstable patient. ${ }^{4}$ Substances are purged using basic physical principles.

\section{Physical Principles}

1. Ultrafiltration: The transport of solvent (the

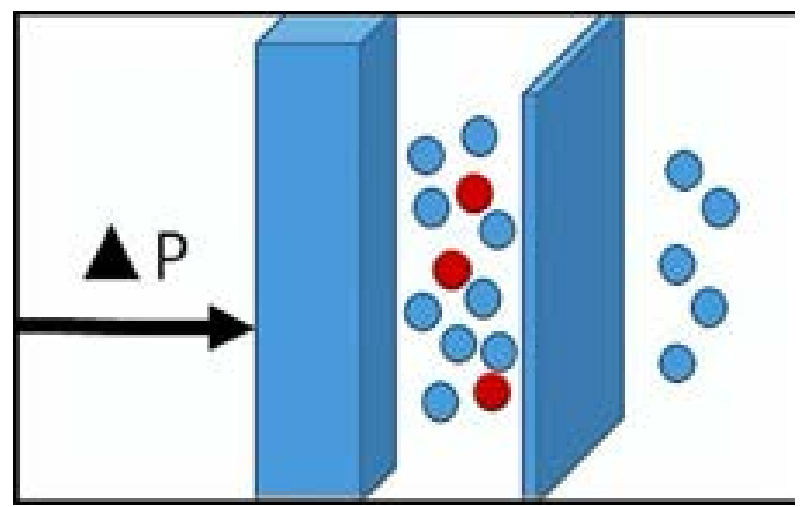

Figure 1: Principle of onvection

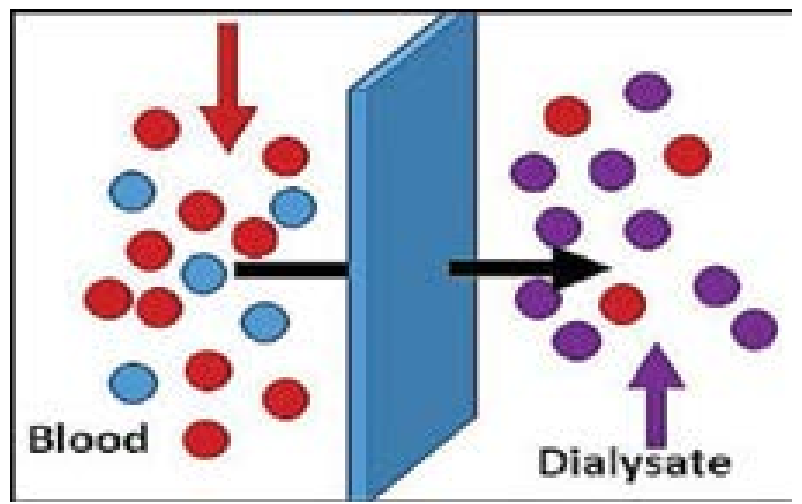

Figure 2: Principle of Diffusion

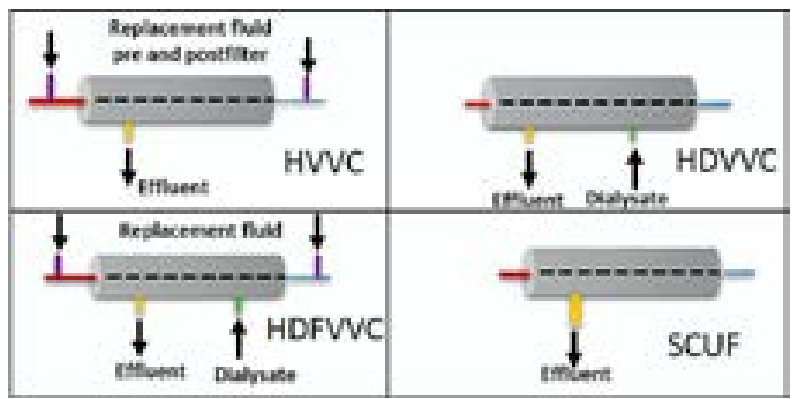

Figure 3: Most frequently used renal replacement therapy modalities in pediatric intensive care. liquid part of plasma) through a semipermeable membrane, generated by a pressure gradient between the blood compartment and the dialysate/ultrafiltrate compartment. It is influenced by the filter's physical properties and the pressure gradient. The volume of ultrafiltrate may be partially or completely replaced. It is a technique which may be used on its own, as in slow continuous ultrafiltration therapies(SCUF), or as part of a hemofiltration or hemodiafiltration therapy.

2. Convection: The movement of a solvent (plasma) through a semipermeable membrane due to a transmembrane pressure gradient, allows the secondary transport of solutes 6 ], in addition to generation of ultrafiltrate. It removes high and medium molecular weight molecules. It is used in continuous venovenous hemofiltration and continuous venovenous hemodiafiltration renal replacement therapies ${ }^{4,5}$ (Figure 1).

3. Diffusion: The removal of solutes through a semipermeable membrane, which is produced by a solute concentration gradient between the blood on one side of the membrane and the electrolyte (dialysis) solution on the other side of the membrane, which moves counter-current to the flow. ${ }^{7}$ Diffusion mainly removes small molecular weight substances but is ineffective in clearing high molecular weight substances. It is used in both intermittent and continuous hemodialysis ${ }^{4}$ (Figure 2).

\section{Modalities of continuous renal replacement therapy}

Different types of renal replacement therapy can be classified based on the various physical properties described, and according to the patient's pathophysiological needs (Figure 3).

1. Slow Continuous Ultrafiltration (SCUF): A technique in which the blood passes through a highly permeable filter, producing an ultrafiltrate which is not replaced, and which corresponds to the patient's weight loss. It is used for controlling fluid overload. It uses pump flow rates $(\mathrm{Qb})$ of $100-250 \mathrm{ml} / \mathrm{min}$ and ultrafiltrate rates (Quf) of $5-15 \mathrm{ml} / \mathrm{min}^{7}$

2. Continuous Venovenous Hemofiltration (CVVH): A technique which uses the physical principle of convection, in which blood is passed through a highly permeable filter producing an ultrafiltrate, and solute purging by drag. The ultrafiltrate produced during passage through the membrane is partially or completely replaced in order to achieve blood purification and 
appropriate intravascular volume management. This replacement may be before the filter (predilution) or after the filter (post-dilution). It uses pump flow rates $(\mathrm{Qb}) \quad 2-6 \mathrm{ml} / \mathrm{kg} / \mathrm{min}$ and an ultrafiltrate flow of $15-60 \mathrm{ml} / \mathrm{min} .^{5-7}$

a. Predilution: Fluid replacement given before the hemofilter. It prevents circuit coagulation and extends the life of the filter. It reportedly reduces treatment efficiency due to the effect of filtrate dilution. ${ }^{4}$

b. Post-dilution: Hemofiltration fluid replacement which is given after the hemofilter. It allows greater purging of solutes with a greater risk of coagulation due to hemoconcentration in the distal part of the membrane..$^{1-4}$

3. Continuous Venovenous Hemodialysis (CVVHD): A technique in which the blood is passed through a low permeability dialyzer in an extracorporeal circuit in venovenous mode, and through a counter current mechanism, to the dialysate compartment in the membrane. It produces solute purging through the principle of diffusion, with molecular movement efficiency inversely proportional to the solutes' molecular weight and size, and directly proportional to their concentration. It uses a Qb of 100-250 ml/ $\mathrm{min}$ and a dialysate flow $(\mathrm{Qd})$ of $15-60 \mathrm{ml} / \mathrm{min} .{ }^{7,8}$

4. Continuous Venovenous Hemodiafiltration (CVVHDF): A technique that utilizes both principles of hemodialysis and hemofiltration. When a blood passes through a semipermeable membrane, pressure across the filter is utilized to drive movement of water (ultrafiltrate) and solutes (through convection) from blood to dialysate compartment. This filtrate is partially or completely replaced as is CVVF. In addition, filter's waste compartment also contains dialysate solution and concentration gradient is used to move solutes from blood to dialysate compartment through diffusion process. ${ }^{7}$

5. Intermittent Hemodialysis: A diffusion technique in which the blood and the dialysate circulate counter- current to each other. It generates molecular transfer at a much higher velocity than continuous renal replacement therapies. ${ }^{1}$ It is ideal in acute fluid overload, intoxications, hyperammonemia, tumor lysis syndrome and metabolic alterations. ${ }^{9}$

6. Hybrid Techniques: A name given to renal replacement therapy modalities which combine the therapeutic advantages of continuous therapies in critical patients with the logistical advantages of intermittent therapies (less need for anticoagulation, less therapy time). They are known as Sustained low-efficiency daily dialysis (SLEDD), Extended daily dialysis with filtration (EDDf), Prolonged daily intermittent renal replacement therapy (PDIRRT), and extended intermittent hemodialysis. They use the same type of machinery but are used for less than 24 hours and for a longer time than intermittent hemodialysis. The pump and dialysate velocities are slower $(100 \mathrm{ml} / \mathrm{min} \text { or } 6 \mathrm{~L} / \mathrm{h})^{7}$

Requirements for carrying out renal replacement therapy:

Some considerations are needed for carrying out RRT:

Vascular Access: There are different types and sizes according to the duration and the patient's size, and the flow produced depends on their radius and length. These are parallel or coaxial double-lumen catheters for central jugular or femoral placement. Subclavian access should be avoided due to the risk of stenosis. ${ }^{8}$ Femoral catheters are used nearly 3 times more frequently in children than internal jugular and subclavian $^{3}$ (Table 1)

Filter or dialyzer: These are biocompatible synthetic membranes made from different material such as polysulfone, polyamide, polymethylmethacrylate, polyethersulfone, and polyarylethersulfone / polyamide. These membranes are set up as an internal chamber through which the blood passes, and an external chamber where the ultrafiltrate passes. Sometimes, blood priming can be necessary in small infants when the extracorporeal volume of circuit exceeds $10-15 \%$ of the patient's estimated blood volume. ${ }^{3}$ The membrane area determines a greater or lesser permeability, mass transfer and ultrafiltration, as well as blood flow to achieve their goal. Therefore, their surface area will depend on the patient's surface area, which must be taken into account for selecting the appropriate size according to the child's size. ${ }^{1}$

Extracorporeal circuit: Is made up of a circuit which holds a blood volume available in three sizes: neonatal $25 \mathrm{ml}$, pediatric $75 \mathrm{ml}$, and adult $125 \mathrm{ml}$. The extracorporeal circuit volume includes the

Table 1: Recommended catheter size according to patient weight

\begin{tabular}{c|c}
\hline Patient's weight & Catheter size \\
\hline $3-6 \mathrm{Kg}$ & 7 French double lumen \\
\hline $6-15 \mathrm{Kg}$ & 8 French double lumen \\
\hline $15-30 \mathrm{Kg}$ & 9 French double lumen \\
\hline$>30 \mathrm{Kg}$ & $10-12.5$ French double lumen \\
\hline
\end{tabular}


dialysate priming volume and the blood volume in the connector. If the volume is greater than $10 \%$ of the whole-body blood volume, blood should be used to prime the circuit. ${ }^{1}$

Pump flow: The dialysis circuit functions as a pump, generating blood flows which depend on the size of the catheter. The smallest ones $(7-8 \mathrm{~F})$ do not generate flows greater than $60-80 \mathrm{ml} / \mathrm{min}$. The recommendation of using $3-10 \mathrm{ml} / \mathrm{kg} / \mathrm{min}$ flows is extrapolated from adult and animal models, and works adequately. In the smallest children, higher flows may be needed in order to generate an adequate return pressure and to help improve the life of the circuit, without affecting solute purging. ${ }^{8}$

Dialysate/replacement flow: Corresponds to the volume of effluent administered during therapy and is calculated as the sum of the fluid removed (ultrafiltrate) plus the replacement flow (Qr) plus the dialysate flow (Qd): $\mathrm{UF}+\mathrm{Qr}+\mathrm{Qd}$, in combined therapies. It is considered to represent the purging of solutes, assuming that filter permeability remains constant. ${ }^{10}$ The dose is taken from adult studies. Ronco et al in 2000 showed that doses above $35 \mathrm{ml} /$ $\mathrm{kg} / \mathrm{h}$ improved survival in adults with acute renal failure, when compared to a dose of $20 \mathrm{ml} / \mathrm{kg}$ / $\mathrm{hr}$. Since then, other studies have evaluated renal replacement therapy doses with mixed results. The VA/NIH ATN study showed that among 1,124 critical adult patients there was no difference in 60 day survival or the need for new therapy between doses of $35.8 \pm 6.4 \mathrm{ml} / \mathrm{kg} / \mathrm{h}$ and $22 \pm 6.1 \mathrm{ml} / \mathrm{kg} / \mathrm{h}$. However, these patients fluctuated between continuous and intermittent therapies, making it less comparable to Ronco's initial study.

Another study, RENAL, which included patients on CVVHDF, showed no differences in survival or need for therapy at 90 and 60 days, respectively, using high vs. low dose intensity, 33.4 and $22 \mathrm{ml} / \mathrm{kg} / \mathrm{h}$ respectively. The consensus of these studies is that there is no difference between the use of therapy at $20-25 \mathrm{ml} / \mathrm{kg} /$ hr. and higher doses. However, there are technical considerations which could lead to not achieving these flows in practice, but rather only $70-80 \%$ of the predicted level, and therefore the consensus is to use doses ranging from $25-40 \mathrm{ml} / \mathrm{kg} / \mathrm{h}$ in diffusion or convection in children. ${ }^{1,8,11}$

Replacement solutions: These are solutions used to achieve volume control in patients treated with hemofiltration or hemodiafiltration techniques. They contain electrolytes and solutes to replace those lost in therapy, bicarbonate-based fluids are considered standard of care in adults and children. ${ }^{8}$

Anticoagulation: The current options for circuit anticoagulation are a continuous infusion of heparin or the use of citrate, the only large observational study in pediatrics demonstrated that heparin and citrate are equally effective with similar circuit lifespans but suggested that bleeding complications were more common with heparin. ${ }^{3}$ Use of citrate should be avoided in liver failure patients, whose metabolism may be erratic. ${ }^{1}$

\section{EFFECTIVENESS OF RENAL REPLACEMENT THERAPIES}

Once any renal replacement therapy is implemented, it is important to monitor and evaluate its processes, and thus measure its effectiveness. Effectiveness will depend both on the characteristics of the extracorporeal circuit as well as on the velocities of flow and replacements which may be achieved and may be evaluated using various indices which are described as follows.

\section{Filter and membrane characteristics}

Evaluate membrane performance through parameters which evaluate its permeability to water and solutes. ${ }^{5}$ These are:

1. Membrane ultrafiltration coefficient (KUF): represents the amount of liquid which moves through the membrane by unit of pressure and area

$\mathrm{KUF}=\mathrm{QUF} / \mathrm{TMP} \times \mathrm{A}$ (Measurement units: $\mathrm{ml} / \mathrm{h} / \mathrm{mmHg} / \mathrm{m}^{2}$ )

Bearing in mind that each fiber in the filter is a cylinder, the cross-sectional area of each fiber will be equal to $2 \pi \times \mathrm{rx}$, where $\mathrm{r}$ is the radius of each cylinder, and $\mathrm{l}$ is its length. If $\mathrm{Nf}$ is the total number of fibers in the filter, the total area will be $2 \times \mathrm{Nf} \times \Pi$ $\mathrm{x}$ rx 1

KUF defines membrane flow according to its hydraulic permeability, but not its solute permeability ${ }^{5}$, as follows:

- Low flow: $<10 \mathrm{ml} / \mathrm{h} / \mathrm{mmHg} / \mathrm{m}^{2}$

- Medium flow: $10-25 \mathrm{ml} / \mathrm{h} / \mathrm{mmHg} / \mathrm{m}^{2}$

- High flow: $>25 \mathrm{ml} / \mathrm{h} / \mathrm{mmHg} / \mathrm{m}^{2}$

2. Mass transfer coefficient (K0A): Represents the membrane capacity to remove solutes through diffusion by area.

It is equal to flux $(\mathrm{Jd})$ times unit of area $(\mathrm{ml} / \mathrm{min})$

$\mathrm{K} 0 \mathrm{~A}=\mathrm{Jd} \times \mathrm{A}$

According to Fick's law of diffusion, Jd is directly proportional to the concentration gradient and diffusion coefficient, and inversely proportional to 
membrane thickness. ${ }^{4}$

$\mathrm{Jd}=\mathrm{D} \times\left(\mathrm{C}_{1}-\mathrm{C}_{2}\right) / \mathrm{d} \mathrm{x}$

Where $\mathrm{D}$ is the diffusion coefficient of the substance, $\mathrm{C}_{1}$ is the initial concentration, $\mathrm{C}_{2}$ is the final concentration, and $\mathrm{d} x$ is the membrane thickness.

3. Sieving coefficient: Is the ratio between the concentration of solute removed in the ultrafiltrate $\left(\mathrm{C}_{\mathrm{UF}}\right)$ over the mean plasma concentration of the substance in the filter. ${ }^{5}$

Where $\mathrm{C}_{\mathrm{pi}}$ and $\mathrm{C}_{\mathrm{pf}}$ are the concentrations of the substance at the entrance and exit of the filter. Practically, this is simplified to:

$\mathrm{SC}=\mathrm{C}_{\mathrm{UF}} / \mathrm{C}_{\mathrm{pi}}$

If this ratio is close to 1 , the substance can diffuse easily between the two compartments.

\section{Pressures within the circuit:}

Filter permeability can be evaluated by monitoring the circuit pressures. Several pressure patterns have been described which are associated with filter malfunction.

If the circuit is not occluded, pressures normally remain constant or close to the initial values, but if the circuit is occluded, there is a change in pressure levels, depending on the cause. ${ }^{12}$

There are five monitoring values for pressure:

1. Arterial or entrance pressure (AP): Its value will always be negative, since it corresponds to the pressure that the pump exerts to extract the patient's blood. It should be kept at about -100 $\mathrm{mmHg}$.

2. Venous return or post filter pressure (VP, $\left.\mathbf{P}_{\text {POST }}\right)$ : Its value will always be positive, and corresponds to the pressure at which the blood returns to the patient. It should be kept at about $100 \mathrm{mmHg}$, and should be lower than the prefilter pressure.

3. Prefilter pressure $\left(\mathbf{P}_{\mathbf{P R E}}\right)$ : Its value should always be positive and greater than the venous or return pressure. It allows us to indirectly evaluate the clotting status of the filter. It should be kept at about 100 to $150 \mathrm{mmHg}$.

4. Effluent or ultrafiltrate pressure: Corresponds to the ultrafiltrate compartment pressure. It is needed for calculating the transmembrane pressure. It may be calculated using the following formula:

$\left.\mathrm{P}_{\mathrm{UF}}=\left(\mathrm{P}_{\mathrm{PRE}}-\mathrm{P}_{\mathrm{POST}}\right) / 2\right)-\mathrm{TMP}$

It is normally positive and greater than $50 \mathrm{mmHg}$. Negative values indicate that the hemofilter produces less than what the ultrafiltrate pump demands, and therefore it must suction to achieve the pre-established objective.

It will depend on the ultrafiltrate flow, the pump velocity, the number of functioning capillaries in the filter, and the type of therapy selected.

5. Transmembrane pressure (TMP): Indicates the difference in pressure on both sides of the membrane, and corresponds to the filter work pressure. It directly controls the ultrafiltrate flow (Quf). It depends on the hydrostatic pressure of the blood compartment $\left(\mathrm{P}_{\mathrm{B}}\right)$, the hydrostatic pressure of the effluent/ultrafiltrate $\left(\mathrm{P}_{\mathrm{D}}\right.$ or $\left.\mathrm{P}_{\mathrm{UF}}\right)$, and the oncotic pressure of the blood $\left(\Pi_{B}\right)$.

The transmembrane pressure may vary throughout its length (1), and therefore its value is an average throughout the filter and does not show the true local pressure in the filter.

$$
\operatorname{TMP}(1)=\mathrm{P}_{\mathrm{B}}(1)-\mathrm{P}_{\mathrm{D}}(1)-\Pi_{\mathrm{B}}(1)
$$

It may be expressed in a simplified form as follows: $\mathrm{TMP}=\Delta \mathrm{PB} / 2-\Delta \mathrm{PD} / 2-\Delta \Pi \mathrm{B} / 2$

$T M P=\left(P_{B} i-P_{B} f / 2\right)-\left(P_{D} i-P_{D} f / 2\right)-(\Pi B i-\Pi B f / 2)$

However, CRRT machines do not measure $\mathrm{P}_{\mathrm{d}} \mathrm{i}$ nor $\Pi В$ directly, and therefore it is estimated using the following formula:

$\mathrm{TMP}=\left(\mathrm{P}_{\mathrm{PRE}}+\mathrm{P}_{\mathrm{POST}} / 2\right)-\mathrm{P}_{\mathrm{UF}}$

It should be kept $<200 \mathrm{mmHg}$. Higher values indicate filter malfunction (a decreased permeability coefficient), since that means that the pump must suction more, (in other words increase $\mathrm{P}_{\mathrm{PRE}}$ ), in order to obtain the same ultrafiltrate. An increase of 1 $\mathrm{mmHg}$ in TMP increases the risk of filter clotting by $1.5 \% .^{13}$

The pressure ratio to generate ultrafiltrate should be as follows:

$P_{B}(1)>P_{D}(1)+\Pi B(1)$

The critical equilibrium point in the filter is achieved when $P_{B}(1)=P_{D}(1)+\Pi B(1)$, and it implies that there is no movement towards either side of the membrane. If, on the other hand, the ratio is inverted: $P_{B}(1)<$ $P_{D}(1)+\Pi B(1)$, dialyzing fluid will pass to the blood (retrograde, or "back filtration").

Now, if VP increases, or AP is more negative, it may possibly be related to vascular access problems, while an increase in $\mathrm{P}_{\mathrm{UF}}$ indicates imminent filter clotting, which follows from changes in $\mathrm{P}_{\text {PRE }}$. The latter may also increase if there is circuit kinking.

If TMP increases, this may be secondary to a high 
Table 2: System Alarms

\begin{tabular}{l|c|c|c}
\hline \multicolumn{4}{|c}{ Priority } \\
Alarms & High & Medium & Low \\
\hline Air detection & $\mathrm{X}$ & & \\
\hline Leakage of blood & $\mathrm{X}$ & & \\
\hline Venous pressure & $\mathrm{X}$ & & \\
\hline Transmembrane pressure & & $\mathrm{X}$ & \\
\hline Arterial pressure & $\mathrm{X}$ & & \\
\hline Ultrafiltrate volume & & $\mathrm{X}$ & \\
\hline Ultrafiltrate rate & & & $\mathrm{X}$ \\
\hline Temperature & & $\mathrm{X}$ & \\
\hline Conductivity & & & $\mathrm{X}$ \\
\hline Water supply & & $\mathrm{X}$ & \\
\hline
\end{tabular}

rate of ultrafiltrate, clot formation in the filter, or catheter malfunction. ${ }^{12}$

\section{Filtration fraction (FF):}

This is the percentage of plasma which is filtered. In other words, the amount of plasma withdrawn (QUF) compared to the total plasma which passes through the filter $(\mathrm{Qp})$.

Experts recommend keeping it below $20 \%$ in order to avoid hemoconcentration and filter clotting. ${ }^{4,14}$

$\mathrm{FF}=\mathrm{QUF} / \mathrm{Qp}$

With $\mathrm{Qp}=(\mathrm{QB} \times(100-\mathrm{Hct}) / 100) \times 100 \mathrm{QP}=\mathrm{QB} \times(1$ $-\mathrm{Hct}$ )

$\mathrm{FF}=\mathrm{QUF} / \mathrm{QB} \times(1-\mathrm{Hct})$

We should keep in mind that if the replacement is carried out pre-filter, the filtration fraction decreases, and therefore prolongs the life of the filter. It should be calculated as follows:

$\mathrm{FF}=\mathrm{QUF} / \mathrm{QB} \times(1-\mathrm{Hct})+\mathrm{QR}$ PRE

In addition, it is useful to know the concentration ratio (CR,) which establishes the degree of hemoconcentration within the filter:

$\mathrm{CR}=\mathrm{QUF} / \mathrm{QB}+\mathrm{QR} \mathrm{PRE}=\mathrm{QR}$ Post $\mathrm{QR} \mathrm{PRE}+$ QUFnet + QR PRE/ QB + QR PRE

\section{Resistance to blood flow (Rb)}

This calculates blood flow resistance through the hollow fibers of the hemofilter. It is calculated using the following formula:

$\mathrm{R}_{\mathrm{b}}=\mathrm{P}_{\mathrm{PRE}}-\mathrm{P}_{\mathrm{POST}} / \mathrm{Q}_{\mathrm{B}}$

A very high-pressure difference at the filter entrance and exit is due to an increased $R$ in the filter, possibly related to clotting. Therefore, it is estimated that it should be $<1.5$, since higher values have been associated with clotting.

\section{PROBLEMS TO BE RESOLVED DURING RRT}

Given that continuous renal replacement therapies are used more and more in intensive and pediatric care units, and they are techniques to be carried out continuously in the intensive care unit, the pediatric intensivist must be familiar not only with the proper ordering for different pathologies, but should also be aware of the clinical problems which could affect the patient's stability with regard to renal replacement therapy, and the technical problems related to the machine which may affect the continuity, safety and effectiveness of therapy (Table 2). Therefore, it is important to monitor circuit pressures and take action when alarms sound. ${ }^{15}$

\section{CONCLUSION}

In conclusion, we consider that slow or continuous renal replacement therapies are a safe and useful therapy, presenting important challenges for clinicians with regard to understanding the therapies, filter and circuit longevity, and, obviously, their effectiveness in reaching the goals established for each patient. It is very important to carry out a strict and continuous monitoring of the previously described variables in order to have a good follow-up and to solve any technical problems that may arise.

Conflict of interest: The authors declare that they have no conflict of interest.

Authors' contribution:

EO, EL, DM- Concept, conduction and write manuscript

JF-Concept, conduction and write manuscript and leader of project 


\section{REFERENCES}

1. Sethi SK, Bunchman T, Raina R, Kher $\mathrm{V}$. Unique considerations in renal replacement therapy in children: Core curriculum 2014. Am J Kidney Dis 2014;63(2):329-45. [PubMed] [Free full text] DOI: 10.1053/i. ajkd.2013.08.018

2. Goldstein SL. Advances in pediatric renal replacement therapyfor acute kidney injury. Semin

Dial. $2011 ; 24$ (2): 187-91. [PubMed] DOI: $\underline{10.1111 / \mathrm{i} .1525-}$ 139X.2011.00834.X

3. Sutherland SM, Alexander SR. Continuous renal replacement therapy in children. Pediatr Nephrol. 2012 Nov;27(11):2007-2011. [PubMed] DOI: $10.1007 / \mathrm{s} 00467-011-2080-\mathrm{x}$

4. Cerdá J, Ronco C. Modalities of continuous renal replacement therapy: Technical and clinical considerations. Semin Dial. 2009;22(2):114-22. [PubMed] DOI: $\underline{10.1111 / 1.1525-}$ 139X.2008.00549.X

5. Neri M, Villa G, Garzotto F, Bagshaw S, Bellomo R, Cerda J, et al. Nomenclature for renal replacement therapy in acute kidney injury: basic principles. Crit Care. 2016;10;20(1):318. [PubMed] [Free full text] DOI: 10.1186/s13054-016-1489-9

6. Tolwani A. Continuous renal-replacement therapy for acute kidney injury. N Engl J Med 2012;367:250514. [PubMed] [Free full text] DOl: 10.1056/NEJMct1206045

7. Ricci Z, Romagnoli S, Ronco C. Renal Replacement Therapy. F1000Res. 2016 Jan 25;5. pii: F1000 Faculty
Rev-103 [PubMed] [Free full text] DOI: 10.12688/f1000research.6935.1

8. Sutherland SM, Alexander SR. The prospective pediatric continuous renal replacement therapy (ppCRRT) registry: a critical appraisal. Pediatr Nephrol. 2014 Nov;29(11):2069-76. [PubMed] DOI: 10.1007/s00467013-2594-5

9. Villa G, Ricci Z, Ronco C. Renal Replacement Therapy. Crit Care Clin. 2015 0ct;31(4):83948. [PubMed] DOI: 10.1016/i. ccc. 2015.06 .015

10. Macedo E, Claure-Del Granado R, Mehta RL. Effluent volume and dialysis dose in CRRT: time for reappraisal. Nat Rev Nephrol. 2011;8(1):5760. [PubMed] DOI: $10.1038 /$ nrneph.2011.172

11. Ronco C, Bellomo R, Homel P, Brendolan $A$, Dan $M$, Piccinni $P$, et al. Effects of different doses in continuous veno-venous haemofiltration on outcomes of acute renal failure: a prospective randomised trial. Lancet. 2000;356(9223):2630. [PubMed] [Free full text] DOI: 10.1016/S0140-6736(00)02430-2

12. Ejaz AA, Komorski RM, Ellis $\mathrm{GH}$, Munjal S. Extracorporeal circuit pressure profiles during continuous venovenous haemofiltration. Nurs Crit Care. 2007;12(2):81-5. [PubMed] DOI: $\underline{10.1111 / \mathrm{j} .1478-}$ 5153.2006.00192.x

13. Kakajiwala $A$, Jemielita $T$, Hughes JZ, Windt K, Denburg M, Goldstein $\mathrm{SL}$, et al. Membrane pressures pre- dict clotting of pediatric continuous renal replacement therapy circuits. Pediatr Nephrol. 2017 Jul;32(7):12511261. [PubMed] [Free full text] DOl: 10.1007/s00467-017-3601-z

14. Ronco C, Ricci Z, De Backer D, Kellum JA, Taccone FS, Joannidis M, et al. Renal replacement therapy in acute kidney injury: controversy and consensus. Crit Care. 2015;19:146. [PubMed] [Free full text] DOI: $\underline{10.1186 / \mathrm{s} 13054-}$ 015-0850-8

15. Tennankore KK, D'Gama C, Faratro R, Fung S, Wong E, Chan CT. Adverse technical events in home hemodialysis. Am J Kidney Dis. 2015;65(1):11621. [PubMed] DOI: 10.1053/i. ajkd.2014.08.013

16. Saha M, Allon M. Diagnosis, Treatment, and Prevention of Hemodialysis Emergencies. Clin $\mathrm{J}$ Am Soc Nephrol. 2017;12(2):357369. [PubMed] [Free full text] DOI: 10.2215/CJN.05260516

17. Santiago MJ, López-Herce J, Urbano J, Solana M, del Castillo J, Ballestero Y, et al. Complications of continuous renal replacement therapy in critically ill children: a prospective observational evaluation study. Crit Care 2009;13(6): R184. [PubMed] [Free full text] DOl: 10.1186/cc8172

18. Prada M, Fernández-Sarmiento J, Rojas A y cols. Regional citrate anticoagulation for continuous renal replacement therapy in children. Pediatr Nephrol. 2017;32:703-711. [PubMed] DOI: $10.1007 / \mathrm{s} 00467-$ 016-3544-9 